\title{
A THEOREM ON RANK WITH APPLICATIONS TO MAPPINGS ON SYMMETRY CLASSES OF TENSORS
}

\author{
BY MARVIN MARCUS ${ }^{1}$
}

Communicated by Gian-Carlo Rota, March 31, 1967

1. Results. Let $R$ be a field containing a real closed subfield $R_{0}$. The main results of this announcement follow.

Theorem 1. Let $A_{1}, A_{2}, \cdots, A_{p}$ be $m \times n$ matrices with entries in an infinite subset $\Omega$ of $R$ containing the natural numbers in $R_{0}$. Let $k$ be a positive integer and assume that the rank of each $A_{i}$ is at least $k$. Then there exist nonsingular matrices $E$ and $F$ with entries in $\Omega$ such that every set of $k$ rows (columns) of $E A_{i} F$ is linearly independent, $i=1, \cdots, p$.

Corollary 1. If the matrices $A_{1}, \cdots, A_{p}$ in Theorem 1 each have rank precisely $k$ then every $k$-square subdeterminant of $E A_{i} F$ is nonzero, $i=1, \cdots, p$.

TheOREM 2. If $A_{1}, \cdots, A_{p}$ are $n$-square complex hermitian matrices all of rank at least $k$ then there exists a nonsingular matrix $E$ such that every set of $k$ rows (columns) of $E^{*} A_{i} E$ is linearly independent.

In 1933, J. Williamson [1] gave necessary and sufficient conditions for the compounds of two matrices to be equal. The nontrivial part of his result states the following: if $A$ is a complex matrix of rank $r$ and $r>m$ then $C_{m}(A)=C_{m}(B)$ if and only if $A=z B$ where $z^{m}=1$. A result closely connected to Theorem 1 and generalizing the Williamson result can be proved. We state our theorem in an invariant setting.

Thus, let $V$ be an $n$-dimensional space over the complex numbers, let $H$ be a subgroup of the symmetric group $S_{m}, m \leqq n$, and let $\chi$ be a complex valued character of degree 1 on $H$. A multilinear function $f\left(v_{1}, \cdots, v_{m}\right)$ is symmetric with respect to $H$ and $\chi$ if $f\left(v_{\sigma(1)}, \cdots, v_{\sigma(m)}\right)$ $=\chi(\sigma) f\left(v_{1}, \cdots, v_{m}\right)$ for all $v_{1}, \cdots, v_{m}$ in $V$ and all $\sigma \in H$. Let $P$ be a vector space and $f$ a fixed multilinear function symmetric with respect to $H$ and $\chi, f: V \times \cdots \times V \rightarrow P$, such that for any multilinear function $g, g: V \times \cdots \times V \rightarrow U$, also symmetric with respect to $H$ and $\chi$, there exists a linear $h: P \rightarrow U$ that makes the following diagram commutative:

1 This research was completed under Grant AFOSR 698-67 awarded by the Air Force Office of Scientific Research. 


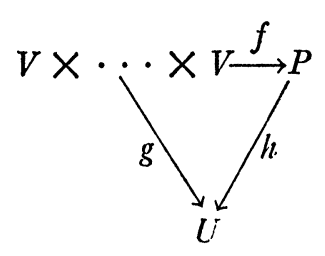

Then the pair $P, f$ is called a symmetry class of tensors associated with $H$ and $\chi$, e.g., $H=S_{m}, \chi=$ sgn, $P=\Lambda^{m} V, f\left(v_{1}, \cdots, v_{m}\right)=v_{1} \Lambda \cdots \wedge v_{m}$, the usual $m$ th Grassmann product. If $T$ is a linear transformation on $V$ then one defines a linear transformation $h$ via the diagram (1) with $U=P, g\left(v_{1}, \cdots, v_{m}\right)=f\left(T v_{1}, \cdots, T v_{m}\right)$. In this case $h$ is called the transformation induced by $T$ and will be denoted here by $K(T)$. If $P=\wedge^{m} V$ then $K(T)$ is the $m$ th compound of $T, C_{m}(T)$. Another example: if $H$ is the identity group then $P=\bigotimes_{i=1}^{m} V$, the $m$ th tensor space over $V$, and $K(T)=\Pi^{m}(T)$, the $m$ th Kronecker power of $T$.

We have the following generalization of Williamson's result to an arbitrary symmetry class of tensors as described above. We do not present a proof here but this generalization depends directly on Theorem 1 for the case $p=2$.

THEOREM 3. If the rank of $T$ is $r$ and $r>m$, then $K(T)=K(S)$ if and only if $T=z S$ where $z^{m}=1$.

CoROllary 2. If $V$ is a unitary space, the rank of $T$ is $r$, and $r>m$, then $T$ is normal if and only if $K(T)$ is normal.

2. Proof outline. We say that a set of $m \times n$ matrices $\left(A_{1}, \cdots, A_{p}\right)$ have property $R_{k}$ if there exists a nonsingular $n$-square matrix $F$ such that every set of $k$ columns of $A_{i} F, i=1, \cdots, p$, is linearly independent: this is abbreviated $\left(A_{1}, \cdots, A_{p}\right) \in R_{k}$. It is clear that if we can prove that any set of $p$ matrices all of rank at least $k$ satisfy $\left(A_{1}, \cdots, A_{p}\right) \in R_{k}$ then Theorem 1 will follow. Observe that if $S_{1}, \cdots, S_{p}$ are nonsingular $m$-square matrices then

$$
\left(S_{1} A_{1}, \cdots, S_{p} A_{p}\right) \in R_{k}
$$

if and only if $\left(A_{1}, \cdots, A_{p}\right) \in R_{k}$.

Now let $L$ be the $n$-square matrix whose $(i, j)$ entry is $i^{j}, i, j$ $=1, \cdots, n$. It is routine to verify that every subdeterminant of every order of $L$ is nonzero. Next, let $t_{1}, \cdots, t_{n}$ be independent indeterminates over $R$ and define an $n$-square matrix $L\left(t_{1}, \cdots, t_{n}\right)$ over $R\left[t_{1}, \cdots, t_{n}\right]$ whose $(i, j)$ entry is $t_{i} i i^{i}, i, j=1, \cdots, n$. It follows that any specialization of $t_{1}, \cdots, t_{n}$ to nonzero elements of $\Omega$ pro- 
duces a matrix every one of whose subdeterminants is nonzero. According to (2) we can take $\left(A_{1}, \cdots, A_{p}\right)=\left(H_{1}, \cdots, H_{p}\right)$ where $H_{i}$ is the Hermite normal form of $A_{i}, i=1, \cdots, p$. Next, consider the matrices $B_{i}=H_{i} L\left(t_{1}, \cdots, t_{n}\right)$ and define the polynomial $p_{i}\left(t_{1}, \cdots, t_{n}\right)$ to be the product of all $C_{n, k}$ entries in the first row of the $k$ th compound matrix of $B_{i}$, i.e., $C_{k}\left(B_{i}\right)=C_{k}\left(H_{i}\right) C_{k}\left(L\left(t_{1}, \cdots, t_{n}\right)\right)$. The fact that $A_{i}$ and hence $H_{i}$ has rank at least $k$ implies that there exists a specialization of $p_{i}$ which is not zero. Hence the polynomial

$$
P\left(t_{1}, \cdots, t_{n}\right)=\prod_{i=1}^{p} p_{i}\left(t_{1}, \cdots, t_{n}\right)
$$

is not zero. It follows from a standard theorem on polynomials that there exist nonzero elements $\xi_{1}, \cdots, \xi_{n}$ in $\Omega$ for which $P\left(\xi_{1}, \cdots, \xi_{n}\right)$ $\neq 0$. In other words, there exist nonzero $\xi_{1}, \ldots, \xi_{n}$ in $\Omega$ for which every entry in the first row of each of $C_{k}\left(H_{i} L\left(\xi_{1}, \ldots, \xi_{n}\right)\right)$ is nonzero, $i=1, \cdots, p$. This means that every set of $k$ columns of each of $H_{i} L\left(\xi_{1}, \cdots, \xi_{n}\right)$ is linearly independent and proves the result.

The rest of the results announced above follow from Theorem 1.

\section{REFERENCE}

1. J. Williamson, Matrices whose sth compounds are equal, Bull. Amer. Math. Soc. 39 (1933), 108-111.

University of California, Santa Barbara 